\title{
An Integrated Control Strategy Adopting Droop Control with Virtual Inductance in Microgrid
}

\author{
Jianjun $\mathrm{Su}^{1}$, Jieyun Zheng ${ }^{2}$, Demin $\mathrm{Cui}^{1}$, Xiaobo $\mathrm{Li}^{1}$, Zhijian $\mathrm{Hu}^{2}$, Chengxue Zhang ${ }^{2}$ \\ ${ }^{1}$ Dezhou Power Supply Company, Shandong Electric Power Group Co., Dezhou, China \\ ${ }^{2}$ School of Electrical Engineering, Wuhan University, Wuhan, China \\ Email: zhijian_hu@163.com
}

Received 2013

\begin{abstract}
As there exists sorts of distributed generators in microgrid, an integrated control strategy containing different control methods against corresponding generators should be applied. The strategy in this paper involves PQ control and droop control methods. The former aims at letting generators like PV output maximum power. The latter stems from inverter parallel technique and applies to controlling generators which can keep the network voltage steady to make the parallel system reach the minimum circulation point. Due to the unworthiness of droop control applied in low-voltage microgrid of which the impedance ratio is rather high, the paper adopts the droop control introducing virtual generator and virtual impedance. Based on theoretical analysis, simulation in Matlab is also implemented to verify the feasibility of the strategy.
\end{abstract}

Keywords: Microgrid; Integrated Control; PQ Control; Droop Control; Virtual Impedance

\section{Introduction}

With the demand for energy expanding and the concern about global warming growing deep, more and more distributed generators including fuel cell, wind power and photovoltaic cell at al. get widely used [1]. Thus we come to a definition of microgrid, namely, a network group consisting of DG units which are on the distribution network side and provide energy for local areas, energy storage devices, and loads [2,3]. Microgrid mainly operates in two modes: grid mode and island mode.

The control mode of microgrid is classified into two: principle-subordinate control and peer-to-peer control. According to this thought, droop control which is based on the droop characteristic of traditional grid is mainly used [4,5].In the practical application of microgrid, nevertheless, there is a diversity of DG units ranging from PV to DG units. Due to this, distinct control methods are designed for each kind of DG units, that's integrated control strategy [6].

Reference [5] introduces virtual impedance, however it doesn't give a detailed description of the realization of this improvement.

This paper not only introduces virtual impedance into the integrated control of microgrid, but also specifies on its detailed realization. Depart from analyzing its feasibility, the paper verifies the strategy by simulation.

\section{Structure of Microgrid Controllers}

As can be seen from Figure 1, the controller of DG is composed of the controller which compounds grid voltage reference and grid current reference and UI tracking controller. The paper will specify on the former.

\section{Integrated Control Strategy of Microgrid}

When microgrid is connected into power distribution network, every DG unit gets controlled with PQ control method as the voltage and the frequency of the system have been adjusted by infinite power grid and it's the most important for DG units to keep power balance among each other. When microgrid is disconnected from power distribution network, there's a need to maintain the amplitude and frequency of the voltage of power grid

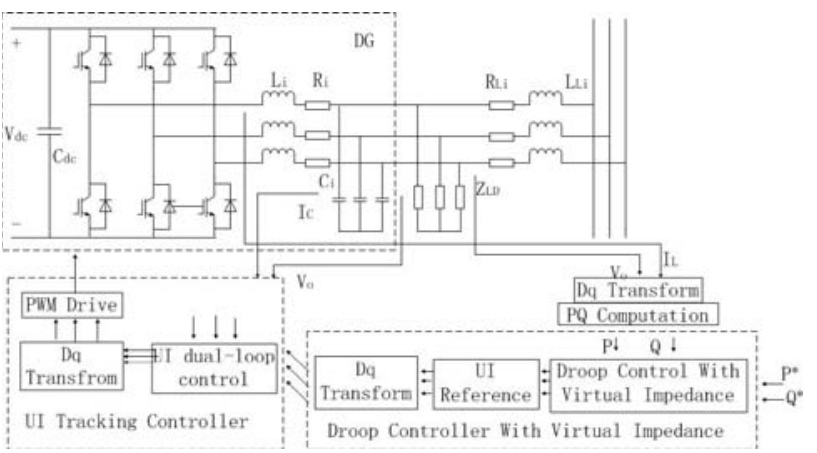

Figure 1. Control diagram of droop control with virtual inductance in Microgrid system. 
As a result, droop control is adopted to provide reference for grid voltage and its frequency.

\subsection{PQ Control Method}

PQ control method is based on the feed-forward decoupling of $\mathrm{dq}$ transformation and realizes maximum power output of DG by adjusting active current and reactive current to track reference current [7].

The equation of reference current is:

$$
\left\{\begin{array}{l}
i_{d r e f}=\frac{P_{r e f}}{U_{d}} \\
i_{\text {qref }}=-\frac{Q_{r e f}}{U_{d}}
\end{array}\right.
$$

Then by feed-forward decoupling of quadrature direct axis current, we get reference for outer loop voltage. That's to make the inverter reach the reference output power using the classical voltage and current dual-loop control.

\subsection{Droop Control Method}

As DG units are connected to PCC with isolating transformers, DC components of their injection currents are no more than 5 percent of rated output currents [8]. The sketch diagram of power transmission of a DG is shown as Figure 2.

Where, Point A is the output point of a DG unit which is consisted of a DG and its filtering system. Point B is the input point of power distribution network. The impedance of the intermediate transmission line is $Z_{L}=R+j X$. Assume that the injected power of point $\mathrm{A}$ is $S=P+j Q$, the output power of the DG can be expressed as follows.

$$
\begin{aligned}
& P=\frac{E_{1}\left[R\left(E_{1}-E_{2} \cos \delta\right)+E_{2} X \sin \delta\right]}{R^{2}+X^{2}} \\
& Q=\frac{E_{1}\left[X\left(E_{1}-E_{2} \cos \delta\right)-E_{2} R \sin \delta\right]}{R^{2}+X^{2}}
\end{aligned}
$$

The feasibility of droop control is based on the line being inductive. The precondition is often satisfied by setting the parameters of the dual-loop controller without virtual impedance [9]. The equivalent output impedance of the inverter is

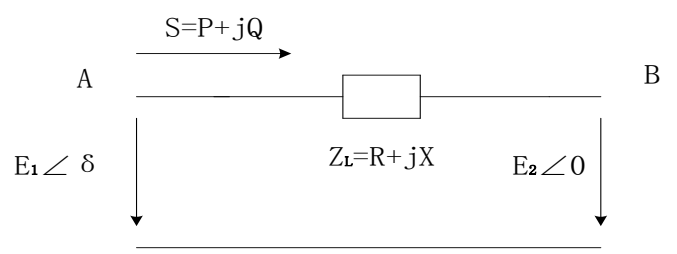

Figure 2. Power transmission diagram.

$$
Z(s)=\frac{L s^{2}}{L C s^{3}+k k_{p w m} C s^{2}+\left(1+k k_{p w m} k_{p}\right) s+k k_{p w m} k_{i}}
$$

where, $\mathrm{k}$ is the scale parameter of current control in dualloop control, $k_{p}$ and $k_{i}$ are the scale parameter and integral parameter of voltage control respectively. $k_{p w m}$ is the magnification coefficient of the inverter and we can take that $k_{p w m}=\frac{V_{d c}}{2}$. We can choose parameters which make $\mathrm{Z}(\mathrm{s})$ inductive in the frequency range of $50 \mathrm{~Hz}$ and the inductive range is not that wide.

\subsection{Droop Control Method with Virtual Impedance}

As microgrid is mostly low voltage network, the impedance ratio of its lines is rather large. From (3), we know that with large impedance ratio, the value of

$E_{1} X\left(E_{1}-E_{2} \cos \delta\right)$ is relatively small, while the influence of $E_{1} E_{2} R \sin \delta$ which is also affected by active power increases.

In the parallel system of DG units, when the output active power differs from each other, it may occur that some DG units absorb reactive power while others release [10]. To solve this problem, we need to decouple $\mathrm{P}$ and Q. One solution is to make use of coordinate transformation [11], but it involves impedance ratio which is difficult to acquire sometimes. One solution is to design parameters of the controller to turn output impedance inductive which nevertheless is at the mercy of the parameter design of dual-loop control. Besides those, the solution of introducing virtual impedance should be the best.

The advanced droop control embodies the thought of equivalent control. According to it, a DG in microgrid is equivalent to a virtual generator with virtual impedance, which is shown in Figure 3.

Reference [5] has specified on the principle and the feasibility of the advanced method. This paper will tell its detailed realization.

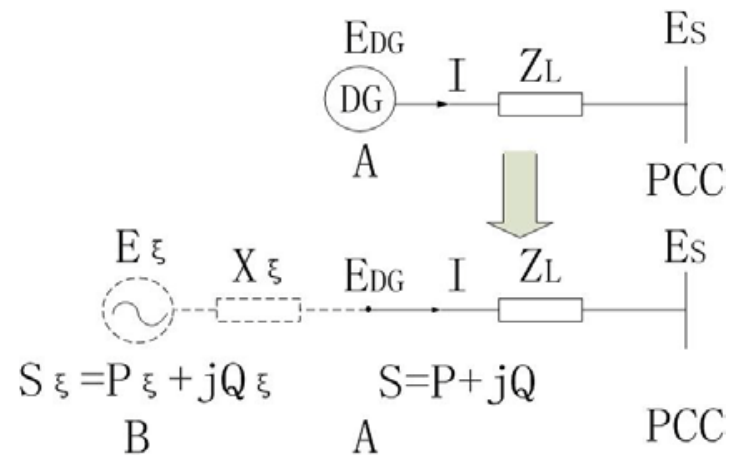

Figure 3. Equivalent Microgrid system with virtual inductance. 


\section{Realization of Advanced Droop Control}

\subsection{Structure of Droop Control with Virtual Impedance}

The control model of droop control is made of three parts: dq transformation and reference power compound, reference voltage and frequency compound, voltage and current dual-loop control. According to equivalent thought, we have

$$
Q=Q_{\xi}-I^{2} X_{\xi}
$$

The instant reference voltage of original DG is

$$
V_{D G}^{*}=e_{\xi}-L_{\xi} \frac{\omega_{C}}{s+\omega_{C}} d i_{D G} / d t
$$

where, $\frac{\omega_{C}}{s+\omega_{C}}$ is the low-pass filter for restraining highfrequency noise in the virtual line.

\subsection{Simulation of Droop Control with Virtual Impedance}

In the power compound module, introduce the output current of DG units. Compound output reactive power $Q_{\xi}$ of the virtual generator with the dq components of the output voltage of DG units. Meanwhile, cross multiply the dq components of output current to get $I^{2}$ and multiply by $X_{\xi}$. On the basis of the logical relation in (6), we get reference reactive power for DG units.

The active power compound module needn't modification as $P=P_{\xi}$. According to (6), on the basis of original dq components of reference voltage, minus respectively the voltage drop of output currents down the virtual impedance and get new reference output voltage. Where, the drop is achieved by dq transforming the output current, going through the differential part and a transfer function of a filter with a filtering capacitor, finally going through a proportional element of $L_{\xi}$.

According to equivalent principle, we have

$$
E_{\xi}=\sqrt{\left(E_{D G}+\frac{Q X_{\xi}}{E_{D G}}\right)^{2}+\left(\frac{P X_{\xi}}{E_{D G}}\right)^{2}} \approx E_{D G}+\frac{Q X_{\xi}}{E_{D G}}
$$

Depending on the thought of averaging, thus we have

$$
E_{\xi}=\frac{1}{2}\left[\left(E_{D G \max }+E_{D G \min }\right)+\left(\frac{Q_{\min }}{E_{D G \max }}+\frac{Q_{\max }}{E_{D G \min }}\right) X_{\xi}\right]
$$

According to parameters in simulation, assume

$$
L_{\xi}=100 \mathrm{mH}, \quad E_{\xi}=567 \mathrm{~V} \text {. }
$$

\section{Simulation Analysis}

Establish a model of a system having 4 paralleled DG units shown in Figure 4.

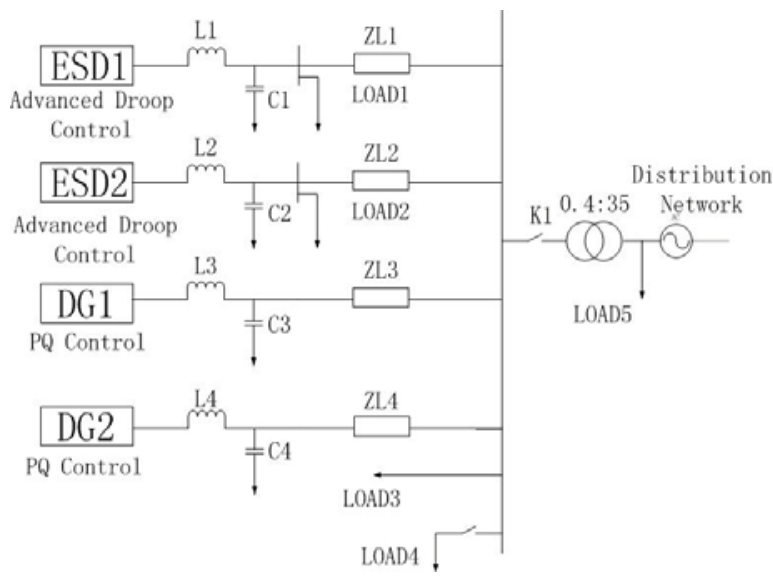

Figure 4. Structure of simulation model.

PV resources are both controlled with PQ control method. Energy storage device (ESD) is both controlled with droop control method and advanced droop control method. The advanced method sets the line between DG and PCC inductive by designing the parameters of the controller, while the original realizes power decouple by introducing virtual impedance.

The voltage of two ESD is $800 \mathrm{~V}$. Their rated power is $1 \mathrm{~kW}$. Reference output voltage is $380 \mathrm{~V}$. Filtering inductance is $50 \mathrm{mH}$ and capacity is $20 \mu \mathrm{F}$. With regard to line impedance, $R_{L}=0.641 \Omega / \mathrm{km}, X_{L}=0.101 \Omega / \mathrm{km}$ and the length is $50 \mathrm{~m}$. The PWM carrier frequency is $6000 \mathrm{~Hz}$.

The action time of switches is: the illumination intensity of PV decreases at $0.167 \mathrm{~s}$, microgrid becomes island mode at $0.3 \mathrm{~s}$, Load 4 is applied at $0.5 \mathrm{~s}$ and cut at $0.8 \mathrm{~s}$, microgrid is again connected with distribution network at 1s. Simulation step: $5 \times 10^{-5} \mathrm{~s}$. Simulation algorithm: ode23. Simulation time: 2 s.

\subsection{Simulation Analysis of Integrated Control}

According to PQ control principle, for droop control, $K_{p}=1, K_{i}=0.5$, as to current control, $K=5$. For PQ control, $K_{p}=155$ and $K_{i}=1$.

Simulation results are as follows. Figure 5(a) and Figure 5(b) are the output voltage of ESD 1 and PV 1 respectively.

As can be seen, in grid mode, due to the effect of the voltage of distribution network, the waveforms are steady and fluctuate little when illumination changes. At $0.3 \mathrm{~s}$ microgrid gets into island mode, the voltage deceases but becomes stable immediately. The amplitude of voltage changes but keeps sine curve at $0.5 \mathrm{~s}$ and $0.8 \mathrm{~s}$ when Load 4 is applied and cut. This suggests that output voltage of DG can be controlled instantaneously to newly become steady with integrated control strategy. The waveforms of microgrid getting into island mode are shown in Figure 6, taking ESD 1 and PV 1 for example. 
The output active power and reactive power of each DG unit is shown in Figure 7. As it can be seen, the power curves of two energy storage device are almost the same and immediately reach a steady value whenever the switches act, which will provide reference voltage for controlling two PV units. As PV2 is close to Load 4, its waveform is a little different from the one of PV1. But they all become steady swiftly, which suggests that PQ control is fit for PV.

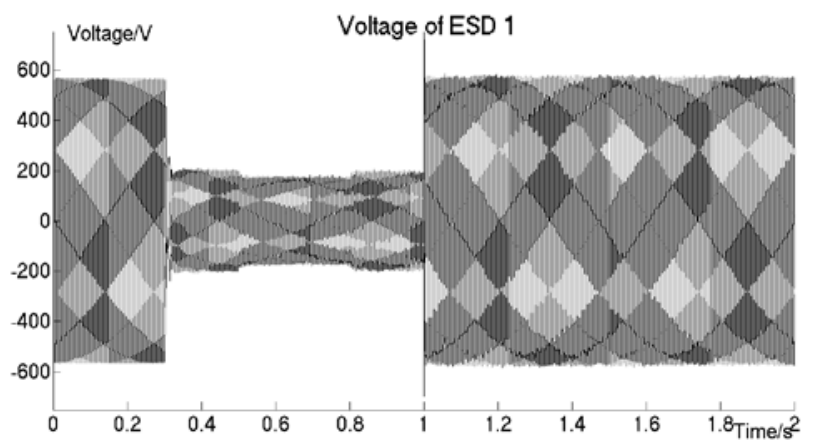

(a)

\subsection{Simulation Analysis of Integrated Control Adopting Advanced Droop Control}

Modify the simulation model according to 3.2. This time, $K_{p}=0.1, K_{i}=0.008$, as to current control, $K=5$. As to PQ control, for PV1, $K_{p}=160$ and $K_{i}=1$. For PV2, $K_{p}=173$ and $K_{i}=1$.

Figure 8(a) and Figure $\mathbf{8 ( b )}$ are the filtered output voltage of ESD 1 and PV 1 respectively.

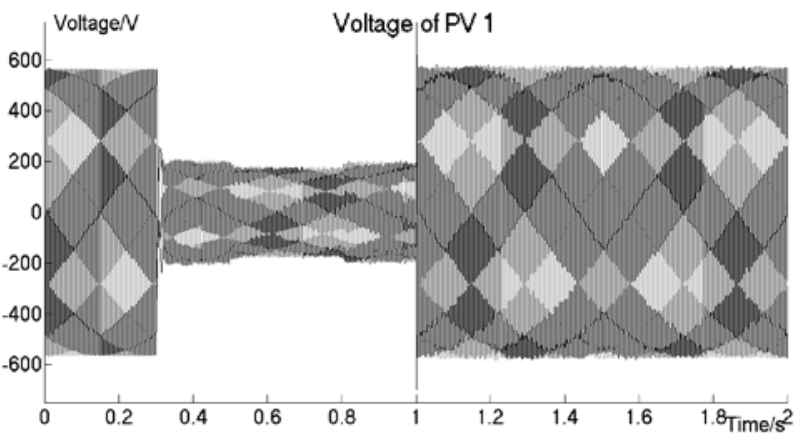

(b)

Figure 5. Output voltage of each distributed generator.

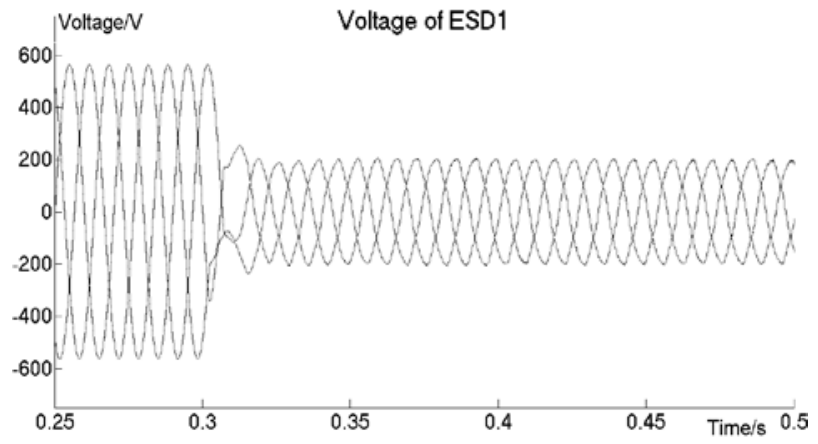

(a)

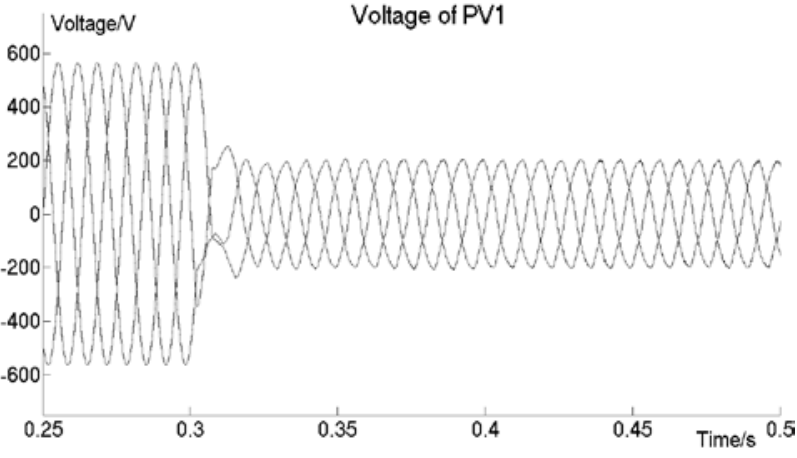

(b)

Figure 6. Waveform of output voltage.

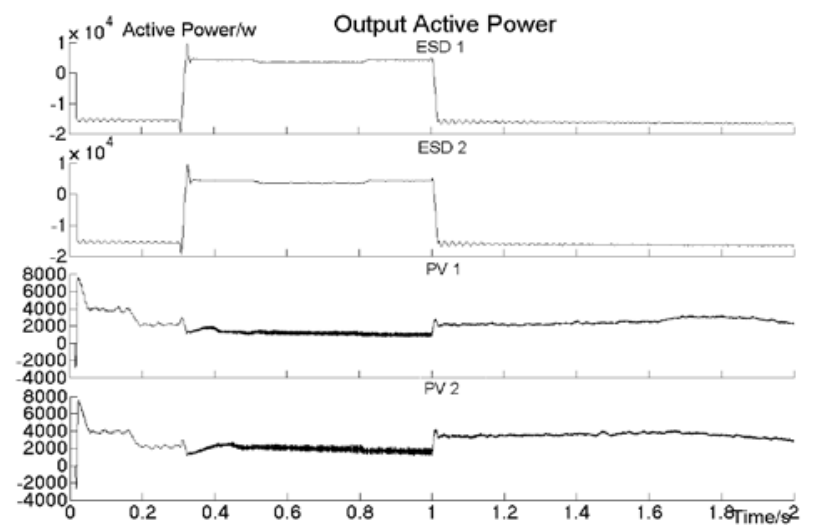

(a)

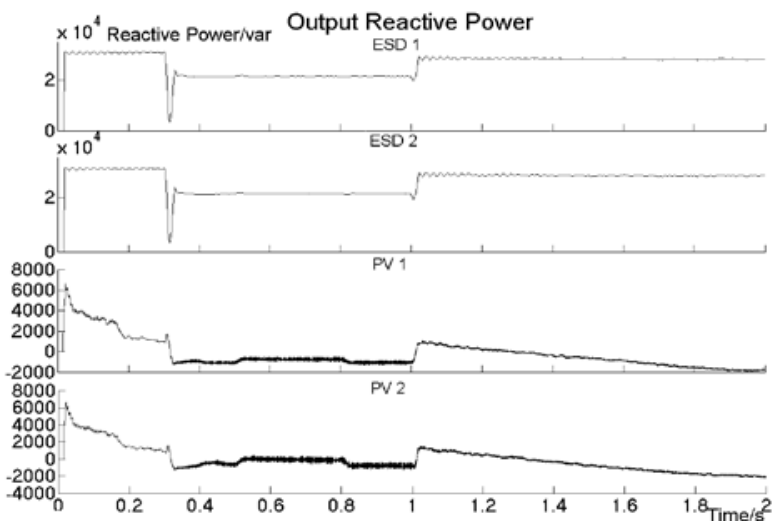

(b)

Figure 7. Output power of each DG. 


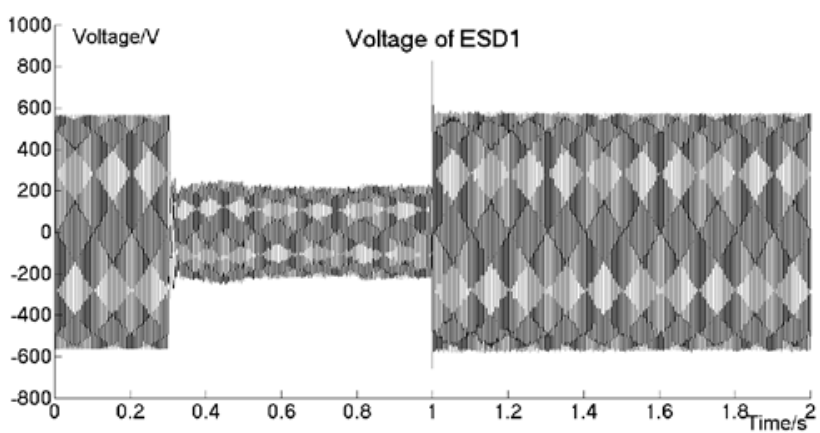

(a)

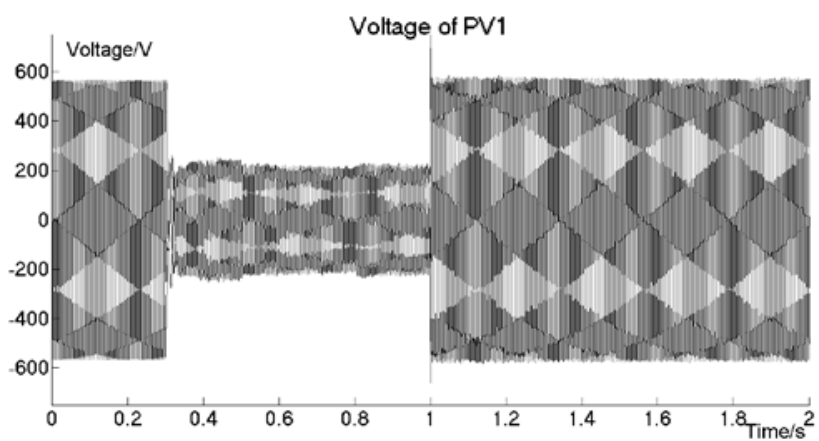

(b)

Figure 8. Output voltage of each distributed generator.

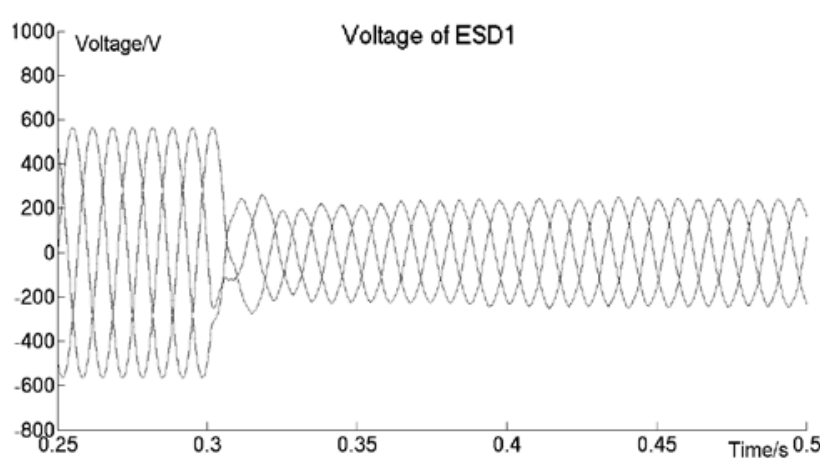

(a)

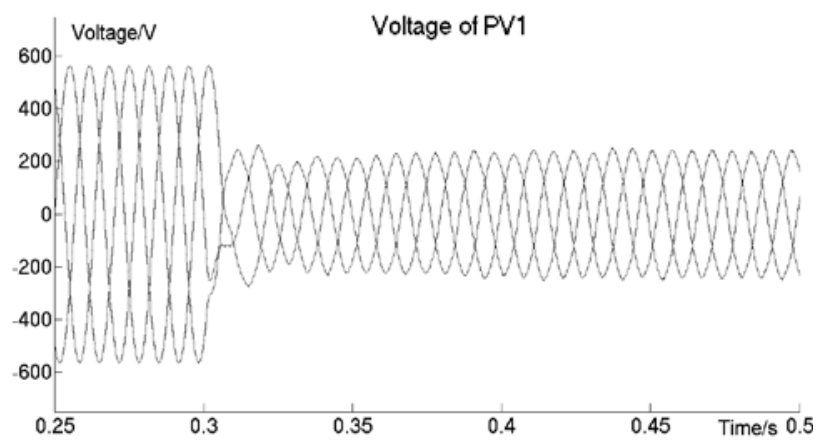

(b)

Figure 9. Waveform of output voltage.

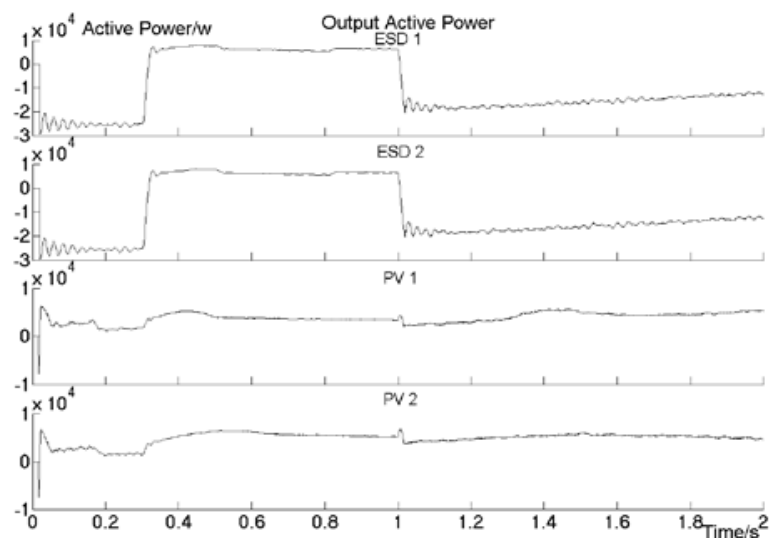

(a)

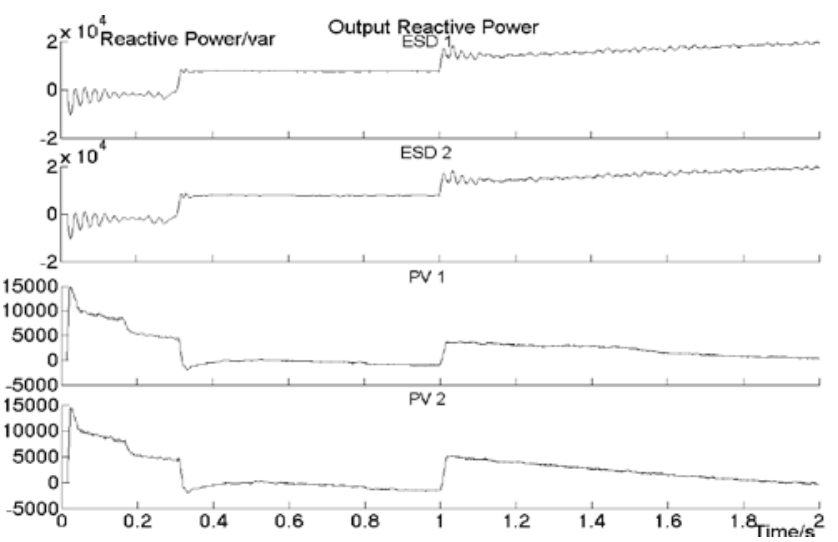

(b)

Figure 10. Output power of each DG.

The adjusting is swift, their waveforms of voltage when switches act are shown in Figure 9. The output active power and reactive power of each DG unit is shown in Figure 10.

With virtual impedance, the power of PV units fluctuates little. Besides, in comparison with the reactive power of original droop control, the fluctuation range of the energy storage device greatly reduces, which indicates that output power is better controlled by integrated control strategy with advanced droop control.

\section{Conclusion}

Upon parallel system of microgrid with energy storage device and $\mathrm{PV}$, this paper makes a study of integrated control strategy depending on characteristics of diverse DG units. What's more, virtual impedance is introduced into droop control to get rid of the restrain of original control on the line impedance. Besides that, specific realization is given in the paper. The results of simulation demonstrates that, the new integrated control strategy can keep output voltage of DG units steady in grid mode and 
island mode, and realize swift transition between the two modes. Furthermore, with virtual impedance, the strategy can better guarantee the stability of output reactive power of each DG units to realize the better decoupling control of active power and reactive power.

\section{Acknowledgements}

This work was financially supported by the Ph.D. Programs Foundation of Ministry of Education of China (20110141110032).

\section{REFERENCES}

[1] J. Huang, C. Jiang, R. Xu. "A review on distributed energy resources and microgrid”, Renewable and Sustainable Energy Reviews, Vol. 12, No. 9, 2008 2465-2476.

[2] W. Saad, Z. Han, H. V. Poor, T. Basar. "Game-theoretic methods for the smart grid: an overview of microgrid systems, demand-side management, and smart grid communications”. IEEE Signal Processing Magazine, Vol. 29, No. 5, 2012, pp. 86-105.

[3] E. Serban, H. Serban. “A control strategy for a distributed power generation microgrid application with voltage-and-current-controlled source converter”. IEEE Transactions on Power Electronics, Vol. 25, No. 12, 2010, pp. 2981-2992.

[4] W. Yang. "Simulation and research of grid connected photovoltaic generation and microgrid operation control”.
Sichuan: Southwest Jiaotong University, 2007.

[5] J. Cheng, S. Li, J. Chen, Z. Wu. "An analysis of decoupling mechanism of droop control using virtual reactance in microgrid”. Automation of Electric Power System, Vol. 36, No, 7, 2012, pp. 27-32.

[6] C. Wang, Z. Xiao, et al. "Intergrated control and analysis of microgrid”. Automation of Electric Power System, Vol. 32, No. 7, 2008, pp. 98-102.

[7] Z. Lu, A. Luo, F. Rong, L. Guo. "Microgrid PQ control strategy analysis under utility voltage imbalance”. Power Electronics, Vol. 44, No. 6, 2010, pp. 71-74.

[8] IEEE 1547-2003 Standard for interconnecting distributed resources with electric power systems. 2003.

[9] Y. Li, C. Kao. “An accurate power control strategy for power-electronics-interfaced distributed generation units operating in a low-voltage multibus microgrid”. IEEE Transactions on Power Electronics, Vol. 24, No. 12, 2009, pp. 2977-2988.

[10] J. Kim, J. M. Guerrero, P. Rodriguez, R. Teodorescu, K. Nam. "Mode adaptive droop control with virtual output impedances for an inverter-based flexible AC microgrid”. IEEE Transactions on Power Electronics, Vol. 26, No.3, 2011, pp. 689-701.

[11] X. Zhou, F. Rong, Z. Lv, S. Peng. "V/f droop-control method with virtual power adopting coordinate rotation in low voltage microgrid”. Automation of Electric Power Systems, Vol. 36, No. 7, 2012, pp. 27-31. 